\title{
SOBRE LA RELACIÓN ENTRE LOS REALISMOS CIENTÍFICO, MODAL Y NOMOLÓGICO*
}

\author{
Bruno Borge** \\ brunojborge@gmail.com
}

RESUMO Os debates sobre o Realismo Cientifico (RC) não só tiveram um começo e um desenvolvimento contemporâneos aos do Realismo Modal (RM) e Realismo Nomológico (RN), mas suas temáticas estiveram também profundamente entrelaçadas. Certa atitude realista em relação às teorias científicas tem sido muitas vezes acompanhada pela adoção de compromissos com a modalidade objetiva e as leis naturais. No entanto, as relações entre estas três posições têm sido pouco exploradas. Neste artigo defendo que manter o RC indiretamente envolve assumir compromissos com o RM e o RN. No primeiro caso, por causa dos argumentos que dão suporte ao $R C$; no segundo, uma vez que, dentro das alternativas disponíveis no debate sobre as leis, o $R N$ é comparativamente melhor para o realista cientifico.

Palavras-chave Realismo Cientifico, Realismo Nomológico, Leis da Natureza, Modalidade, Realismo Modal.

ABSTRACT Debates on scientific realism (SR) not only had a beginning and contemporary developments before those on Modal Realism (MR) and on Nomological Realism (NR), also their thematics were deeply entangled. Some

* El presente trabajo fue realizado con el apoyo de la Agencia Nacional de Promoción Científica y Tecnológica (ANPCyT) y la Universidad de Buenos Aires (UBA). Quisiera expresar mi agradecimiento hacia un evaluador anónimo de "Kriterion" por sus sugerencias y comentarios, que permitieron mejorar la versión final de este artículo.

** Universidad de Buenos Aires. Artículo recibido en 07/01/2016 e aprobado en 04/03/2016.

KRITERION, Belo Horizonte, nº 135, Dez./2016, p. 619-639 
realist attitude regarding other scientific theories has many times been followed by the adoption of commitments with an objective modality and the natural laws. Nevertheless, the relations among these three positions have been little explored. In this article I hold that keeping SR indirectly means committing to $M R$ and NR. In the first case, because of the arguments supporting SR, in the second case, once within the options available in the debate on the laws, NR is - by comparison - better for the scientific realism.

Keywords Scientific Realism, Nomological Realism, Laws of Nature, Modality, Modal Realism.

\section{Introducción}

Mucho se ha dicho en las últimas décadas acerca del Realismo Científico (RC). Sus defensores y detractores han cultivado toda una batería de complejos y poderosos argumentos desplegados por múltiples frentes de análisis filosófico (metafísico, epistémico, semántico). La gran riqueza que el debate entre realistas y antirrealistas científicos fue cobrando le ha valido un lugar entre los tópicos de mayor interés para la filosofía de la ciencia contemporánea. Buena parte de esa importancia se ha debido a la gran variedad de posiciones que procuraron abordar la disputa desde nuevas perspectivas. En particular desde el flanco realista, no sólo se multiplicaron las defensas del RC de corte más bien tradicional, combinando de modo novedoso la variedad de matices que éste ofrece, sino que se instituyeron nuevas posiciones como el Realismo de Entidades y el Realismo Estructural. Con todo, las múltiples batallas gravitaron siempre en torno al foco de la problemática del RC: la existencia de las entidades inobservables postuladas por nuestras mejores teorías científicas.

Contemporáneos al surgimiento de los argumentos más influyentes en el debate Realismo/Antirrealismo Científicos -i.e., los llamados Argumento del No-Milagro y Meta Inducción Pesimista-, en las décadas del '70 y '80 del pasado siglo dos nuevos campos de discusión fueron conformándose. Por una parte, los trabajos de Putnam (1975a) y Kripke (1972) revitalizaron las discusiones en torno al estatus de los enunciados modales. Una nueva teoría de la referencia permitía cruces entre categorías epistémicas y metafísicas que otrora se creyeran excluyentes. ${ }^{1}$ La conmoción que estos argumentos produjeron en el ámbito

1 En sentido estricto, la nueva teoría de la referencia requería además de la doctrina del esencialismo para mostrar la existencia de enunciados necesarios, pero a posteriori, tal como lo ha hecho notar Soames (2006). 
de la filosofía del lenguaje fue en buena parte responsable de que se gestara un creciente interés sobre la naturaleza ontológica del tipo de necesidad que caracterizaba a los enunciados modales. Mientras que para algunos todo el arco de expresiones modales remite a un mismo tipo de necesidad, para otros es necesario distinguir más de una clase de modalidad a fin de caracterizar cada familia de enunciados. En esta última línea se abrieron reflexiones sobre un tipo específico de modalidad correspondiente a los enunciados que describen regularidades naturales, i.e., la necesidad natural. Otros, no obstante, enfrentaron al Realismo Modal (RM) refugiándose en posiciones que heredaban del empirismo humeano el escepticismo respecto de cualquier modalidad de re. ${ }^{2}$ Pero paralelamente, y muy influenciados por los desarrollos en semántica y metafísica modal, algunos filósofos (e.g. Lewis, Carroll, Armstrong) comenzaron a trabajar específicamente sobre el tipo de necesidad característica de enunciados acerca de regularidades naturales, a fin de clarificar el estatus ontológico de las leyes de la naturaleza. De ese modo, tomaron la posta en la carrera por la solución de un problema que había preocupado profundamente-entre otros-a los miembros del Círculo de Viena: la distinción entre leyes y generalizaciones accidentales. El nuevo enfoque, sin embargo, priorizaba las cuestiones metafísicas respecto de la necesidad natural o nomológica, relegando las consideraciones lógicosintácticas sobre los enunciados legaliformes que habían dominado los análisis del Empirismo Lógico. De ese modo, en torno a los argumentos que en un principio pretendían tan sólo elucidar el concepto de necesidad natural, fue constituyéndose toda una polémica respecto de la caracterización metafísica de las leyes de la naturaleza. Estas disquisiciones abarcaron un amplio espectro de cuestiones, desde la relación entre la ley y sus instancias o la distinción ley natural/ley científica, hasta problemáticas clásicas como la inducción o la explicación científica. De ese modo, el campo de debate fue complejizándose lo suficiente como para admitir al menos dos distinciones importantes. En primer lugar, y en gran medida como resultado de un posicionamiento respecto de las

2 En algunos contextos ha sido común emplear la expresión 'Realismo Modal' para referir a la posición defendida por Lewis, según la cual la verdad de los enunciados modales se sustenta en una pluralidad de mundos posibles metafísicamente reales. Mi uso aquí es diferente: refiere a toda teoría que postule la existencia de modalidades de re, i.e., de conexiones necesarias en la naturaleza, irreductibles a la mera regularidad o una pluralidad de mundos posibles. En otras palabras, el RM, según lo entiendo aquí, implica un compromiso con hechos intrínsecamente modales. Como queda de manifiesto y veremos en detalle luego, Lewis cae por fuera de esta caracterización. Por simplicidad, y a falta de una expresión mejor, utilizaré 'Realismo Modal' en este último sentido, haciendo una referencia específica a la propuesta de Lewis allí donde fuese necesario. No obstante, esta forma de emplear la mencionada expresión no es infrecuente en la literatura contemporánea (véanse, por ejemplo, Nora Berenstain y James Ladyman (2012), Monton y van Fraassen (2003)). Agradezco a un evaluador anónimo de "Kriterion" el señalarme la necesidad de esta aclaración. 
cuestiones modales y semánticas ya mencionadas, el debate acerca de las leyes dividió a los necesitaristas de los regularistas; por otra parte, incluso muchos de los que admitían la existencia de modalidad objetiva fueron reticentes a aceptar a las leyes como parte su ontología, así, los defensores del Realismo Nomológico (RN) fueron cerrando filas contra las distintas formas que el antirrealismo respecto de las leyes iba tomando.

Los debates en torno al RC no sólo han tenido un inicio y desarrollo contemporáneos a los del RM y el RN, sus temáticas han estado además profundamente imbricadas. La actitud realista respecto de las teorías científicas frecuentemente ha estado acompañada por la aceptación del RM; del mismo modo, ha sido usual definir al RC como la creencia de que las teorías reflejan (al menos aproximadamente) las leyes naturales. Sin embargo, los múltiples cruces entre estos campos de discusión han adolecido en muchos casos del uso de un discurso laxo o poco sistemático que motivó frecuentes confusiones y solapamientos entre posiciones. Ciertas formas de RM, por ejemplo, han sido tenidas por la afirmación del RN, mientras que la aceptación del $\mathrm{RN}$ se ha tomado muchas veces como una condición necesaria y suficiente para suscribir el RC. En otros casos, la verdadera naturaleza de las relaciones entre estas tres posiciones ha sido simplemente inexplorada.

En el presente trabajo me propongo clarificar las relaciones entre el RC (en su versión estándar), el RM y el RN. Sostengo que suscribir el RC implica indirectamente asumir compromisos con el RM y el RN. En el primer caso, en razón de los argumentos que sostienen el RC, en el segundo, en virtud de que, dentro de las alternativas disponibles en el debate acerca de las leyes, el $\mathrm{RN}$ es comparativamente preferible para el realista científico. A tales fines, en la sección 2, presento las tesis y variedades fundamentales del RM y el RN, procurando además dar cuenta de los vínculos entre ambas posiciones. En la sección 3 expongo sumariamente los lineamientos de la versión estándar del RC que será considerada en este trabajo, para luego explorar sus relaciones con el RM. Muestro que, si bien no existe una implicación fuerte del RC hacia el RM en cuanto a sus tesis constituyentes, una implicación más débil puede ser detectada tanto considerando los argumentos principales a favor del RC (3.1) como una de las motivaciones centrales de su formulación, a saber, la de dar cuanta del carácter continuo y acumulativo del conocimiento científico a lo largo del cambio teórico (3.2). La sección 4 está dedicada a esclarecer los vínculos entre $\mathrm{RC}$ y $\mathrm{RN}$. Pese a que el RC no implica el RN, sostengo que, para el defensor del RC, la adopción del RN es comparativamente preferible respecto de otras alternativas disponibles en el escenario del debate. Por último, la sección 5 incluye algunos comentarios finales y las conclusiones de este trabajo. 


\section{Realismo Modal y Realismo Nomológico}

La modalidad, como casi todo en filosofía, puede decirse de muchas maneras. Hay, efectivamente, muchos modos de expresar la necesidad, posibilidad o imposibilidad de que algo ocurra. El problema radica en saber si existen circunstancias que justifiquen la adscripción de valores de verdad a los enunciados que expresan modalidad. Pocos discutirían que esto puede hacerse cuando se trata de enunciados que expresan una necesidad de dicto, como lo hacen los enunciados de identidad, o más en general las llamadas verdades lógicas. El punto conflictivo es si esa adscripción de valores veritativos puede replicarse en enunciados que no sean verdades lógicas. Los candidatos por antonomasia para una respuesta positiva han sido tradicionalmente los enunciados analíticos, i.e., aquellos que resultan verdaderos en virtud de significados. Si bien esta posibilidad ha recibido sendas críticas -tal vez la más resonante de ellas debida a Quine (1951) - la distinción analítico/sintético ha continuado siendo un tema vigente de discusión. En cualquier caso, la tradición identificó a la familia de enunciados necesariamente verdaderos con aquellos cuya verdad puede ser conocida a priori, lo que resulta natural si se concibe el carácter necesario de toda proposición como idéntico o derivado de la necesidad lógica. Pero los trabajos de Kripke y Putnam cuestionaron seriamente dicha identificación. Su teoría de la referencia directa tiene como consecuencia que ciertos enunciados pueden ser necesarios, pero a posteriori. Eso es posible en tanto algunos términos son considerados designadores rígidos, es decir, términos que refieren a la misma entidad en todo mundo posible en los que dicha entidad exista. Los nombres propios, indéxicos como 'ahora' o 'yo' e incluso términos de clase natural son rígidos. De ese modo, un enunciado como 'el agua es $\mathrm{H}_{2} \mathrm{O}$ ' es necesario, en tanto es verdadero en todo mundo posible ('agua' designa siempre aquello que es $\mathrm{H}_{2} \mathrm{O}$, y si algo no es $\mathrm{H}_{2} \mathrm{O}$ entonces no es agua), pero, claramente es $a$ posteriori, dado que sólo una investigación empírica puede traer esa verdad a nuestro conocimiento. La posibilidad de que las categorías de a priori y necesario no fueran coextensivas motivó la sospecha de que la aprioricidad fuese el caso límite de un tipo particular de necesidad: mientras que algunas proposiciones son metafisicamente necesarias, otras resultan epistémicamente necesarias. La polémica acerca de si ambas categorías se reducen o no a un mismo tipo de necesidad (usualmente descripta como monismo vs. dualismo modales) abrió el juego a una nueva cuestión que ya resonaba en ámbitos vecinos a la filosofía del lenguaje, a saber, la de si existe algún tipo de necesidad característica de aquellos enunciados que expresan leyes de la naturaleza, es decir, una necesidad nomológica o natural. Dos puntos en particular hacen de esta distinción un tema espinoso. En primer lugar, la cuestión ya no es meramente lógica ni semántica, 
y escapa incluso del esencialismo que muchos asocian de modo indisoluble a la teoría kipkeana de la referencia. Se trata ahora de si a los enunciados que describen regularidades de nuestro mundo les corresponde un tipo especial de necesidad anclada a la naturaleza. En segundo término, debe determinarse si esa necesidad los hace verdaderos o bien en este, o bien en todo mundo posible. El primer punto constituye la principal razón de la creciente importancia de los debates metafísicos por sobre las consideraciones lógico-semánticas, tanto en el campo del RM como del RN, el segundo es el nudo de la discusión acerca de si la necesidad nomológica es distinta de la metafísica. En general, la polémica viene dada entre aquellos que creen que la necesidad nomológica es idéntica a la metafísica ${ }^{3}$ y quienes postulan a la necesidad nomológica como un tipo independiente de modalidad. ${ }^{4}$

Mi propia mirada sobre el asunto es la siguiente : si bien toda la discusión está atada a la metafísica subyacente que se adopte y a algunas definiciones previas, creo que hay más de una manera plausible de caracterizar ambos tipos de necesidad de modo que constituyan dominios absolutamente independientes. Por el contrario, la identificación total o parcial de la necesidad nomológica con la metafísica presenta algunos inconvenientes de importancia. Si acordamos en que nomológicamente necesario significa algo así como ser verdadero en virtud de las leyes de la naturaleza, es claro que hay ciertos enunciados metafísicamente necesarios que no parecen cumplir con nuestra definición. "Este escritorio es de madera" es un enunciado metafísicamente necesario - no sería este mismo escritorio si fuese de metal- y sin embargo su verdad no parece desprenderse de ninguna ley natural. Por otro lado, la noción de 'mundo próximo' permite dar cuenta de enunciados nomológicamente necesarios en el mundo actual y en mundos posibles próximos, que no son, sin embargo, verdaderos en todo mundo posible. En cualquier caso, la cuestión del monismo, dualismo o, cabe decir, pluralismo modales resulta en lo esencial independiente de la relación entre el RM y el RN, y por tanto del foco de este trabajo. En este marco será suficiente considerar realista modal a quien se comprometa con un correlato objetivo que fundamente la atribución de valores de verdad a enunciados modales, fundamentalmente, a aquellos que se adscriben necesidad/posibilidad a regularidades o fenómenos naturales.

Una salvedad importante en este sentido es que el compromiso del RM no es meramente con una modalidad objetiva, sino con una modalidad objetiva irreductible. Como ya se ha hecho notar, Lewis rechaza la existencia de 
conexiones necesarias en la naturaleza. Sin embargo, postula una pluralidad de mundos posibles metafísicamente reales como hacedores de verdad de los enunciados modales. Es decir, la modalidad es reducida a la existencia de mundos posibles. Psillos (2014), por su parte, ha procurado reducir la modalidad objetiva a la mera regularidad, argumentando que las conjunciones constantes (y no las conexiones necesarias) en la naturaleza son suficientes para la atribución de valores veritativos a los enunciados modales. Más allá de considerar que esa reducción es imposible -entre otras razones porque colapsa en el antirrealismo modal defendido por van Fraassen-, me apegaré aquí a la caracterización del RM como el compromiso con modalidades irreductibles de re.

En una disputa conceptualmente no muy distante, las aproximaciones más tempranas al problema de las leyes de la naturaleza ${ }^{5}$ se enfocaron en las cuestiones lógico-sintácticas que pudieran fundamentar la distinción entre auténticas leyes y meras generalizaciones accidentales. El debate contemporáneo, por el contrario, asume una forma más compleja que admite variados matices. El punto central consiste en determinar si existe un sustrato objetivo que funcione como hacedor de verdad de las leyes formuladas por la ciencia, haciendo de las regularidades que describen algo necesario, y no meramente una feliz coincidencia cósmica. Puede notarse en este punto la importante influencia que los debates en torno a la modalidad de re que acabamos de abordar tuvieron sobre el desarrollo de la cuestión de las leyes. En efecto, algunos han encontrado en las leyes de la naturaleza -es decir, no en los enunciados formulados por la ciencia, sino en un rasgo del mundo mismo- el sustrato objetivo que garantiza la verdad del discurso modal sobre el mundo natural. Las leyes no son meras proyecciones de nuestra creatividad, sino algo en el mundo, son cosas que descubrimos como soporte de la necesidad objetiva de las regularidades, es decir, como su principio tanto explicativo como ontológico.

Para entender apropiadamente la variedad de posiciones enfrentadas, dos importantes distinciones deben ser trazadas entre ellas. La primera diferencia a los regularistas de los necesitaristas, la segunda a los realistas de los antirrealistas nomológicos.

La primera de estas distinciones recoge la bandera de toda una tradición de interpretación de la filosofía de Hume, caracterizada por un marcado escepticismo respecto de la existencia de causas y conexiones necesarias en la naturaleza. Para quienes sostienen posiciones en esta línea, el mundo está constituido por eventos discretos, cada uno de los cuales se autocontiene y es tanto ontológica 
como causalmente independiente del resto, una suerte de mosaico cuyas piezas son estos eventos discretos y causalmente inertes. Muchas de esas piezas se presentan en relaciones regulares de semejanza y contigüidad espaciotemporal, pero fuera de ellas ninguna relación de necesidad las conecta. A decir de Lewis, sólo eso es nuestro mundo, una cosita y luego otra (1986: ix). La analogía es pertinente en un aspecto más: la independencia de cada una de las piezas no implica que no haya regularidades; por el contrario, con la dedicación suficiente podemos encontrar diversos y complejos patrones en la distribución de las piezas, podemos descubrir que toda pieza roja es contigua de una hexagonal, o que a cada grupo de piezas semejantes en color le sigue un número de piezas verdes determinable por alguna función, etc. El punto es que ninguna de esas regularidades identificadas por la investigación empírica está determinada por una necesidad interna (de una pieza implicando a otra) ni externa (del sistema total implicando alguna distribución).

El necesitarismo, por su parte, no debe ser identificado sin más con el RM. Si bien los compromisos modales están implícitos en esta posición, el foco está puesto aquí en brindar un relato positivo acerca del fundamento de tales compromisos. Muchas han sido las variaciones de ese relato, universales, esencias, poderes y disposiciones fueron invocados como el soporte ontológico de los patrones naturales que escapan a la contingencia. Los argumentos en este sentido han seguido una estructura relativamente constante, aduciendo que todo un conjunto de rasgos de nuestro mundo que remiten a la regularidad (la posibilidad de realizar inducciones, de sostener enunciados contrafácticos, de realizar predicciones e incluso de la actividad científica como tal) quedaría inexplicado si no se presumiera la existencia de un fundamento para la necesidad natural.

Esto nos permite abordar la segunda distinción relevante en el debate. Sólo aquellos necesitaristas para quienes el fundamento de la regularidad sean las leyes defienden un RN. El Antirrealismo Nomológico (AN), por tanto, incluye todo el arco de posiciones regularistas y aquellos necesitaristas para quienes las conexiones necesarias en la naturaleza están fundadas en alguna cosa diferente a las leyes. La adhesión a esta forma de AN suele incluir la adopción de una metafísica disposicionalista: ${ }^{6}$ los poderes y disposiciones dotan al mundo de la efectividad causal que se requiere para fundamentar ontológicamente las regularidades. 
Para el RN, entonces, el mundo contiene no sólo conexiones necesarias entre propiedades, sino leyes que las sustentan. La naturaleza de esas leyes ha sido esclarecida de modos diferentes. Para la concepción internalista de Ellis (2001) las leyes existen, pero son internas a sus instancias, más precisamente son las propiedades esenciales de las clases naturales. Otra alternativa es adoptar la concepción externalista de las leyes defendida por Dretske (1977), Tooley (1977) y Armstrong (1983) (de aquí en más DTA). La DTA sostiene que las leyes son un tipo de universal de segundo orden; según su geografía metafísica, las propiedades deben ser entendidas como universales efectivamente instanciados que mantienen entre sí diversas relaciones de necesitación; esas relaciones de necesitación son las leyes. Si tener la propiedad F implica tener la propiedad $\mathrm{G}$, eso se debe a que $\mathrm{F}$ y $\mathrm{G}$ están vinculados en una relación $\mathrm{N}$ de necesidad tal que N(F,G). Teniendo en cuenta ambas concepciones, Mumford (2004) propone dos requisitos básicos que una teoría acerca de las leyes debe cumplir a fin de enrolarse en el RN:

(a) Las leyes deben ser entendidas como una "adición de ser".

(b) Las leyes deben gobernar sus instancias.

La primera implica que cualquiera que decida ser realista acerca de las leyes debe comprometerse con el hecho de que éstas tienen algún tipo de espesor ontológico, debe creer que son cosas en el mundo, una categoría ontológica per se. El segundo supuesto implica que el rol de gobierno sobre sus instancias es no sólo es una característica constitutiva de las leyes mismas, sino un requisito para la viabilidad de una metafísica que las postule.

Pese al carácter mayormente preparatorio de esta sección, sostengo que dos conclusiones relevantes pueden ser esbozadas. En primer lugar, dado el modo en que definimos a las posiciones que aquí están en juego, el RN implica al $\mathrm{RM}$, esto siempre que se piense que el segundo conlleva un compromiso con una modalidad irreductible, tal como aquí ha sido presentado (las propuestas que pretenden reducir la modalidad objetiva a rasgos no modales del mundo -e.g. meras conjunciones constantes- caen por fuera del RM).

En segundo término, debe notarse que la conversa no se cumple: la adopción de compromisos modales no es condición suficiente para suscribir el RN. Ser necesitarista no implica asumir que existen cosas tales como leyes en la naturaleza; por el contrario, pueden tenerse compromisos modales sustentados metafísicamente en poderes y disposiciones inmanentes a las propiedades que constituyen el mundo natural. Alguien podría argüir que un compromiso tal es equivalente al del $\mathrm{RN}$; sin embargo, ha de reparase que la propuesta 
disposicionalista no cumple los requisitos (a)-(b) consignados más arriba, en tanto los poderes y disposiciones no representan ninguna adición de ser ni gobiernan a sus instancias, sino que son internos y autogobernantes.

\section{Realismo Científico y Realismo Modal}

El RC es, fundamentalmente, la tesis que afirma que las entidades inobservables postuladas por nuestras mejores teorías científicas realmente existen. Como ya he mencionado, esta afirmación metafísica tiene también una contraparte epistémica y una semántica, a saber, las afirmaciones de que tenemos conocimiento acerca de esas entidades y que nuestras teorías son capaces de comportar verdad o falsedad - esto último como opuesto al instrumentalismo-. De ese modo, el RC admite diferentes matices según el tipo de compromiso que se adopte respecto a esas dimensiones de análisis. Como el foco de este trabajo será el RC estándar, se asume de aquí en más un compromiso completo con los niveles correspondientes a las tres afirmaciones mencionadas. Dicho compromiso coincide con los matices que toda caracterización estándar omite sin remedio, con posiciones realistas como las de Boyd (1983), Psillos (1999), Niiniluoto (1999) y Chakravartty (2007), entre muchos otros.

Con esto en mente, el objetivo de la presente sección es dar cuenta de la relación entre el RC y el RM.

Un punto no conclusivo, pero no por ello sin importancia, reside en lo que puede denominarse una actitud realista hacia la modalidad, que es típica entre los defensores del RC. Aquellos que creemos que entidades tales como los átomos existen, tendemos a creer que algunas cosas les suceden necesariamente. Es harto común sostener que las teorías científicas nos proveen de auténtico conocimiento en la medida en que alcanzan a 'capturar' la estructura modal del mundo. ${ }^{7}$ Una motivación adicional para esta actitud realista es el hecho de que en el discurso científico comúnmente abundan las referencias a procesos causales, probabilidad objetiva y equilibrio causal; por otra parte, la mera enunciación de muchas leyes científicas sugiere que algunos compromisos modales son inmanentes a la práctica científica. ${ }^{8}$ Pero a pesar de ello, debe reconocerse que el RM no se sigue lógicamente del RC. Constituye una posición perfectamente coherente el sostener que las entidades inobservables postuladas por nuestras mejores teorías existen, negando al unísono la modalidad objetiva. Esa es, de hecho, la posición que Psillos defiende.

7 Véase, por ejemplo, Armstrong (1983); Ladyman (1998); Chakravartty (2007).

8 Para un desarrollo detallado de este último punto, véase Berenstain y Ladyman (2012, p. 152). 
Sostengo, sin embargo, que hay un apoyo conceptual fuerte para la mencionada actitud realista hacia la modalidad. Dicho apoyo no proviene de la formulación del RC, sino más bien de los argumentos en los que los realistas sustentan su posición. Si ese es el caso, el defensor del RC que niegue la modalidad objetiva se encuentra en una posición más débil para sostener su compromiso realista que aquel que sostiene el RM. Ofrezco dos argumentos que ilustran este punto. El primero se vincula con una de las principales armas a favor del RC, el Argumento del No-Milagro (ANM); el segundo se relaciona con el objetivo de encontrar una teoría del significado apropiada para el RC.

\subsection{No-Milagro y explicación metafísica}

Como es bien sabido, el ANM es la mayor fuente de soporte racional para el RC. Su formulación seminal es debida a Putnam, para quien el RC "es la única filosofía que no hace del éxito de la ciencia un milagro" (Putnam, 1975b, p. 73). La manera usual en que este argumento se ha interpretado lo considera como una instancia de la Inferencia a la Mejor Explicación (IME): la verdad (aproximada) de nuestras teorías en la mejor explicación del éxito predictivo de la ciencia.

El ANM ha sido largamente discutido y reconstruido de múltiples modos, ${ }^{9}$ pero puede afirmarse en general que existe una importante laguna en la literatura en cuanto al concepto de explicación que subyace a su formulación, situación que se replica incluso en las discusiones específicas acerca de la IME como forma de inferencia. ${ }^{10} \mathrm{Si}$ bien dicha polémica excede por mucho los límites de este trabajo, creo que es posible de todos modos precisar algunas consecuencias para el problema que nos ocupa. Sostengo que la adopción del AMN impone al realista la adopción de ciertos compromisos modales en dos sentidos. En primer lugar, en virtud de la forma que la explicación metacientífica del éxito de la ciencia toma; en segundo término, por el contenido propio de esa explicación y las nociones que pretende articular.

La apelación a explicaciones metacientíficas del éxito predictivo de las teorías descarta algunas nociones modalmente deflacionarias de explicación. El Modelo de Cobertura Legal -originalmente concebido a partir de un acercamiento humeano al problema de la regularidad- no es adecuado en este contexto, a menos que se esté dispuesto a hablar de algo así como 'leyes metacientíficas'. Y aún si ese fuera el caso habría todavía que elucidar la cuestión de por qué esas

9 Para un panorama general, véase Leader (2013). Algunos de los desarrollos y críticas más recientes son analizados en Borge (2015).

10 Psillos (2002) constituye una excepción. Para él es deseable que la IME pueda acomodarse a distintos conceptos de explicación. 
'leyes' no son meras generalizaciones accidentales. De igual modo, el enfoque mecanisista de Salmon (1984) o el modelo probabilístico de explicación de Railton (1981) no resultan apropiados, fundamentalmente por el hecho de que ésta es una explicación filosófica y no una científica. La naturaleza de la explicación requerida se asemeja a lo que suele denominarse 'explicación metafísica' ${ }^{11}$ la verdad de nuestras teorías ha de conectarse de modo no accidental, sino necesario con el éxito empírico para que constituya una auténtica (de hecho, la mejor) explicación de ese fenómeno. Es en esta misma vena que Lewis sostiene que "explicar [metafísicamente] un evento es brindar cierta información acerca de su historia causal" (1986, p. 218), y de ese modo lo que una explicación metafísica muestra es que a la luz del explanans aquello que se describe en el explanandum "no hubiese ocurrido, o hubiera sido mucho menos probable de lo que en efecto fue" (1986, p. 214). Es precisamente este sentido de explicación metafísica el que captura la relación entre la verdad aproximada de las teorías y el éxito predictivo de la ciencia, relegando al rango de milagrosas a otras explicaciones alternativas. ${ }^{12}$

Pero aun si el mero uso del concepto de explicación en este contexto no se juzgase suficiente para vincular el ANM con los compromisos modales, hay, en segundo término, una razón adicional en el contenido de la explicación invocada. En efecto, la explicación concreta que el realista ofrece para el éxito predictivo postula la verdad (al menos aproximada) de las teorías actuales. Ahora bien, como ya he procurado destacar, esas teorías incluyen todo un bagaje de conceptos modales, especialmente en la formulación de sus leyes. Enunciados como "nada puede viajar más rápido que la luz" son constitutivos de las teorías aceptadas, por lo que predicar verdad de ellas implica sostener prima facie que hay un correlato real que funciona como su hacedor de verdad. Esta intuición de primera mano ha sido cuestionada por quienes defienden una posición regularista respecto de las leyes naturales. El punto central de esa defensa ${ }^{13}$ es que la mera ocurrencia regular de ciertos eventos es suficiente para garantizar la verdad de los enunciados liguliformes. El problema con esta respuesta es que incluso si fuese correcta en el marco de la caracterización de las leyes creo firmemente que no lo es- resulta inadecuada en el contexto especifico del

11 Para una interesante distinción entre explicación metafísica y explicación en metafísica, véase Persson (2011).

12 Es preciso destacar que lo dicho no presupone que el argumento es adecuado, ni en general como forma de inferencia ampliativa, ni en particular como estrategia para justificar los compromisos realistas. El punto sobre el que quiero llamar la atención es simplemente que la forma y contenido del ANM conllevan compromisos modales para quien esté dispuesto a aceptarlo.

13 Véase, por ejemplo, Psillos (2014). 
ANM. Cabe recordar que tal como Swartz (1985) señala, llevado a su límite el regularismo afirma que la ocurrencia regular de los fenómenos no es diferente de una gran coincidencia cósmica, la uniformidad de la naturaleza no comporta ningún tipo de necesidad inmanente. Esa idea encierra en realidad la esencia de toda forma que esta posición puede tomar: la regularidad es un mero hecho, simplemente ocurre que las cosas resulten del modo que resultan. Ahora bien, la función de adscribir verdad a las teorías científicas es precisamente la de ir más allá del mero hecho del éxito empírico, más allá de que simplemente ocurre que nuestras teorías son exitosas en sus predicciones observacionales -esa es, después de todo, la alternativa 'milagrosa'-. De este modo, la única adición que el ANM en conjunción con una concepción deflacionaria de la explicación pareciera aportar es el compromiso metafísico con las entidades inobservables. Pero en ese caso, la ganancia de cambiar la afirmación "simplemente ocurre que nuestras teorías son exitosas respecto de los fenómenos" por la que sostiene que "nuestras teorías son exitosas porque simplemente ocurre que ciertas entidades inobservables se comportan regularmente" resulta, a mi criterio, demasiado escasa como para justificar el compromiso realista. Finalmente, la explicación última del éxito predictivo se desplaza del milagro a la coincidencia cósmica. Los compromisos modales, por tanto, son esenciales para dar al ANM la fuerza que su formulación pretende tener.

\subsection{Descriptivismo Causal: referencia y continuidad del conocimiento}

Dar cuenta del carácter acumulativo y continuo del conocimiento científico a lo largo del cambio teórico es uno de los objetivos centrales de toda forma de $\mathrm{RC}$, fundamentalmente porque ello permite brindar respuestas a las alegaciones de inconmensurabilidad y a la Meta Inducción Pesimista. Nuevas versiones del realismo han procurado encontrar el sustento de dicha continuidad en factores estructurales o matemáticos que eluden consideraciones acerca del significado y referencia de los términos teóricos. Estos proyectos comparten un diagnóstico que señala que las formas de RC estándar-aquellas que son objeto de análisis de este trabajo- han fracasado en su intento de reconstruir la historia de la ciencia en clave realista armados tan sólo con herramientas semánticas heredadas de las teorías clásicas de la referencia. Los defensores del RC estándar, por el contrario, afirman que es posible construir un relato adecuado de la referencia de los términos teóricos, coherente con los presupuestos del realismo.

En este marco, una segunda línea de argumentación permite reforzar el punto central de esta sección. Sostengo que posibilidad de brindar una adecuada teoría de la referencia para el RC descansa en la adopción de compromisos propios del RM. 
Es un hecho ya ampliamente reconocido que tanto las teorías del significado puramente descriptivistas como puramente causales son insuficientes para dar cuenta de la referencia de los términos teóricos en el marco del RC. ${ }^{14}$ Por una parte, ocurre que un cambio de teoría implica que muchos de los términos teóricos son abandonados, y aquellos que se mantienen cambian su sentido (i.e., cambian las descripciones teóricas que les son asociadas), por lo tanto, de acuerdo a los fundamentos del descriptivismo, cambia también su referencia. Ello resulta en la imposibilidad de caracterizar al conocimiento científico como continuo y acumulativo. La teoría causal pura, en cambio, prescinde de descripciones teóricas para caracterizar la fijación de la referencia, pero lo hace a expensas de convertir en trivial el éxito referencial: dado que un término refiere a aquella entidad responsable de los fenómenos que motivaron su postulación, nada impide afirmar que, por ejemplo, 'flogisto' refiere al oxígeno. Quien primero vio una salida a este problema fue Lewis (1984), en el contexto del análisis del argumento modelo-teórico de Putnam: el único modo de anclar la referencia de los términos teóricos coherente con las intuiciones realistas es una teoría híbrida que combine las descripciones inherentes a la introducción de un término teórico con un elemento causal. Esta teoría híbrida es usualmente denominada Descriptivismo Causal. ${ }^{15}$ Es importante notar que el sentido en el que esta posición es 'causal' es doble en el caso específico de los términos teóricos: tal como en la versión estándar de la teoría causal, hay una cadena causal de transmisión de la referencia a partir del acto de bautismo, pero, además, el carácter inobservable de las entidades teóricas requiere de la afirmación de que el referente pretendido es causalmente responsable de los fenómenos que motivaron la introducción del término (Psillos, 2012, p. 222). Más allá de las inflexiones particulares que ha tomado en la obra de cada autor, estos son los lineamientos estándar para esta posición mixta a la luz de las limitaciones de las alternativas puras. Como podrá intuirse, esta posición abre las puertas a toda una variedad de compromisos modales. Para ser breve, me limitaré a listar algunos aspectos del Descriptivismo Causal que sugieren tales compromisos:

- la transmisión de la referencia entre los hablantes es causal y puede rastrearse.

- los fenómenos regulares que motivan la introducción de un término teórico son causados por ciertas entidades inobservables, en virtud de ciertas propiedades o poderes causales.

- esas entidades constituyen una clase natural, frecuentemente caracterizada partir de ciertas propiedades esenciales. 
- el elemento causal concerniente a la fijación de la referencia de dichas clases naturales inobservables es frecuentemente entendido como la designación rígida de dicha clase -i.e., en todo mundo posible donde dich a clase exista.

- ese mismo fenómeno puede ser elucidado en términos de situaciones contrafácticas que funcionan como hacedores de verdad de ciertas afirmaciones modales acerca de la referencia.

Ciertamente el defensor del RC estándar puede refugiarse en la posibilidad de buscar una alternativa fuera del Descriptivismo Causal. Esto, sin embargo, presenta dos dificultades: en primer lugar, debe prescindir de la que es sin dudas la teoría de la referencia más aceptada por los realistas; en segundo término, la presunta alternativa ha de toparse con el hecho (señalado por Lewis) de que las puras descripciones, sin la adición de un elemento causal, no resultan suficientes para anclar la referencia de los términos teóricos.

\section{Realismo Científico y Realismo Nomológico}

Es preciso comenzar esta sección dejando claro el siguiente punto: el RC no implica el RN. Tampoco los argumentos usualmente usados para sustentar las creencias realistas se vinculan de modo relevante con los compromisos nomológicos. Nada indica que afirmar el RC tenga como consecuencia -sea directa o por medio de argumentos asociados-sostener la existencia de entidades tales como leyes. El sentido en que las consideraciones siguientes pueden ser interpretadas en favor de concluir que existe una relación positiva entre $\mathrm{RC}$ y $\mathrm{RN}$ es mucho más moderado. Sostengo que la posición que resulta de afirmar conjuntamente el RC y el RN es comparativamente más fuerte que aquellas que, de modos diversos, afirman el RC sin comprometerse metafísicamente con las leyes de la naturaleza.

La defensa de ese punto requiere de algunas distinciones. En primer lugar, es preciso detallar tres actitudes que condensan las diferentes reacciones que desde el RC se han tenido contra el RN.

(a) No hay leyes naturales, tampoco conexiones necesarias en la naturaleza, sólo regularidades.

(b) No hay conexiones necesarias en la naturaleza. Tampoco hay leyes en el sentido propio del RN, pero sí en uno deflacionario: son los axiomas del mejor sistema que describe la naturaleza.

(c) No hay leyes, pero sí conexiones necesarias en la naturaleza. 
El enfoque (a) apuesta a prescindir totalmente de la distinción entre leyes y generalizaciones accidentales, bajo el supuesto general de que tal distinción no es significativa en la práctica científica ni en nuestra compresión filosófica de ella, aun bajo los límites del realismo. Esta mirada es subsidiaria, por una parte, de la creciente tendencia (encanada principalmente por la llamada Escuela de Stanford) a señalar que las leyes científicas más generales son abstracciones e idealizaciones que guardan poca o nula relación con los fenómenos que las instancian, o con las entidades inobservables que los causan. Por otra parte, se inscribe en la (más antigua) tradición inaugurada por Russell, que juzga que el concepto de causalidad es dispensable para la ciencia, y perjudicial para la filosofía. ${ }^{16}$ El enfoque (b), por el contrario, rescata el valor de la distinción entre leyes y meras generalizaciones, pero, a diferencia del RN, pretende establecerla epistémicamente, sin la apelación a relaciones objetivas de necesitación. El modo más corriente de cristalizar esta idea es la Teoría de los Mejores Sistemas (TMS), que señala que las auténticas leyes son aquellos enunciados que figuran como axiomas o teoremas en el mejor sistema explicativo que la ciencia pueda producir. ${ }^{17}$ Por último, el enfoque (c) sostiene que la mejor explicación de la regularidad natural no postula leyes que gobiernen los fenómenos, sino poderes causales inmanentes a las propiedades físicas de los objetos. De ese modo, postula un fundamento explicativo para el discurso científico acerca de la regularidad, pero uno distinto del ofrecido por el $\mathrm{RN}$.

Cada una de estas propuestas para desterrar el $\mathrm{RN}$ de los compromisos realistas presenta algunos problemas. La primera de ellas ha de enfrentar el hecho de que (como ya he señalado) tanto el discurso científico como los análisis filosóficos que lo han tenido como su objeto están plagados de referencias a la modalidad en general, y a relaciones causales en particular. Además, debe arreglárselas para explicar por qué la distinción presuntamente infundada entre leyes y generalizaciones accidentales parece tener el estatus de una intuición de sentido común. ${ }^{18}$ Por último, resta la cuestión no menor de la relación entre las leyes, la modalidad objetiva y la explicación científica. Una de las motivaciones centrales para adoptar el RC consiste en su caracterización de las teorías como brindando explicaciones de los fenómenos naturales. En ese sentido, los abordajes tradicionales de la explicación científica (y buena parte de los no tradicionales) la vinculan estrechamente los conceptos de ley y causalidad. Un

16 En Price y Corry (2007) puede hallarse una rica discusión en torno a esta tradición.

17 Defensas de esa posición pueden encontrarse en Lewis (1973); Loewer (1996); Psillos (1999).

18 Creo que es importante no soslayar el hecho de que toda la discusión en torno al estatus epistémico y metafísico de las leyes de la naturaleza fue motivada de modo decisivo (y de hecho lo es hoy día) por la necesidad de brindar una justificación filosófica de la mencionada distinción. 
RC que prescinda de ellos se ve en una posición debilitada para dar cuenta de esa característica central del compromiso realista. El segundo enfoque, por su parte, es permeable a todo un abanico de críticas. Primero, la mera noción de Mejor Sistema encuentra un obstáculo en el problema de la subdeterminación de la teoría por los datos. El sistema explicativo ideal tiene presuntamente infinitos equivalentes empíricos, por lo que la TMS debe complementarse con un relato acerca de las virtudes no empíricas que permiten señalar a uno de ellos como el mejor sistema. Las dificultades inherentes a la formulación de dicho relato son también problemas para la TMS. Por otra parte, la fundamentación meramente epistemológica de la distinción entre leyes y generalizaciones accidentales parece despojar al discurso filosófico sobre las leyes de un aspecto tradicionalmente considerado central, como es el carácter explicativo de la noción de ley.

Más allá de todas estas dificultades propias de sus formulaciones particulares, los enfoques (a) y (b) comparten un rasgo común que agrega una dimensión problemática: ambos implican un antirrealismo modal. Los argumentos que presenté en la sección precedente tienen como consecuencia que el rechazo del RM pone al realista científico en una situación discursivamente débil, en tanto los principales argumentos para sustentar su posición requieren de compromisos modales. Si ese es el caso, la plausibilidad del primer y segundo enfoque se ve socavada por su antirrealismo modal.

La tercera propuesta, (c), no adolece de dicho problema, en tanto el rechazo de los compromisos nomológicos se conjuga con la afirmación de conexiones necesarias en la naturaleza. La forma que esta estrategia ha tomado en el debate actual es la de una metafísica disposicionalista: la postulación de poderes causales permite dar cuenta de la regularidad natural sin apelar a compromisos nomológicos. Molnar (1996), Mumford (2004) y Cartwright (1983) son los principales exponentes de esta posición. El paso final de mi argumentación será mostrar que esta metafísica disposicionalista tiene ciertas dificultades que en principio le restan plausibilidad, y cuyas soluciones viables conducen a formulaciones muy cercanas al RN. Antes, sin embargo, quisiera detenerme en dos aclaraciones que pueden relativizar parcialmente los alcances de mis conclusiones.

En primer lugar, el disposicionalismo puede no ser la única alternativa para sostener conjuntamente el RC y el RM rechazando el RN. Sin embargo, es, con todo, la única disponible según los términos del debate contemporáneo, por lo que las puertas para una nueva propuesta en esa línea no están del todo cerradas. Segundo, el RN en sus distintas versiones no carece de problemas en su formulación y articulación. Si bien creo que una articulación completa de dicha posición es posible, debe quedar claro que su defensa no es el objetivo de este trabajo. 
Volviendo entonces a los problemas propios del disposicionalismo, es preciso destacar que éste está comprometido principalmente con propiedades causales. Su apuesta, en pocas palabras, consiste en concebir la modalidad objetiva como inmanente a las propiedades y no como impuesta por leyes de la naturaleza. En este relato los objetos son concebidos como meros agregados de propiedades causalmente efectivas. Estas propiedades casuales, a su vez, son caracterizadas como meros agregados de poderes o disposiciones. Estas disposiciones permanecen en estado de meras potencias hasta que (por la acción de otros poderes) llegan a actualizarse. El punto central es que las disposiciones son metafísicamente reales incluso antes de efectivizarse: un cristal es frágil incluso cuando nunca nada actualice esa disposición. Los problemas para esta posición surgen cuando comenzamos a cuestionar la naturaleza de estas disposiciones. El más significativo es, a mi entender, la cuestión de su identidad. Si la identidad de una disposición no puede reducirse a sus manifestaciones -dado que por hipótesis puede permanecer como no-manifestada- la salida natural parece ser apelar a una esencia particular o quidditas, es decir, a una naturaleza metafísica independiente del perfil causal de la disposición. El problema con esta solución es que una de las principales razones para rechazar la visión categórica de las propiedades asociada al RN es precisamente la imputación de que esta última conduce al quidditismo. El disposicionalismo parece encontrase entonces en un callejón sin salida.

El único de sus defensores que reconoce explícitamente el problema es Mumford. Su solución, sin embargo, dista de ser satisfactoria. En su opinión, la identidad de una disposición debe caracterizarse a partir del hecho de que ésta está dirigida hacia un universal, a saber, el universal correspondiente a la propiedad que instanciaría si se manifestara (Mumford, 2004, p.194). Esta maniobra tiene una desventaja de primera mano: la ardua naturaleza de la relación de estar dirigido hacia, que es determinante para la caracterización de lo que constituye una disposición real. Según es presentada, no se trata de la habitual relación de instanciación entre universales y particulares, ni una de participación. Tampoco puede tratarse en sí misma de una relación causal en tanto la manifestación es por hipótesis condicional.

Un segundo y más acuciante problema deviene de la necesidad de apelar a universales, incluso a universales no instanciados. Una de las motivaciones para el disposicionalismo era la posibilidad de fundamentar la estructura modal del mundo a partir de las propiedades casuales mismas, sin tener que postular entidades "externas" que sirvan de sustento para dicha modalidad. Peor aún, la forma más típica de RN que el disposicionalismo pretende impugnar se basa justamente en la postulación de universales que son el fundamento metafísico último de la efectividad causal. Por lo tanto, bajo esta solución la principal 
ventaja de esta posición se ve opacada: el disposicionalismo acaba con una ontología inflacionaria que requiere de disposiciones, universales instanciados y no instanciados y una misteriosa relación de dirección hacia un universal, i.e., de mucho más de lo que exige la forma más metafísicamente saturada del RN.

De ese modo, a la hora de afirmar el RC, todas las variedades de RN presentan (al menos) la ventaja de implicar compromisos metafísicos más modestos que la alternativa disposicionalista.

\section{Conclusión}

El RC constituye una posición filosófica con límites y tesis precisas acerca del tipo y grado de compromisos que debemos asumir respecto de las teorías científicas, pero es también una cierta actitud que muchos decidimos tomar para emprender una evaluación epistémica de la práctica científica y sus resultados. Esta actitud realista evidencia cierta tendencia a valorar compromisos respecto de la modalidad e incluso de las leyes naturales. Éstos son objetos de defensas y cuestionamientos independientes, pero no es claro, en general, cuál es su relación precisa con el RC. El presente trabajo pretende llenar esa brecha, mostrando que la tendencia implícita en la actitud realista hacia las teorías está justificada. Como hemos visto, si bien el RC no implica lógicamente realismos respecto de la modalidad y las leyes, existen razones indirectas para afirmar un tipo más débil de implicación. El principal argumento a favor del RC y su teoría de la referencia más aceptada determinan un compromiso con el RM. Si bien en un sentido algo diferente, la adopción del RN se ve también favorecida a partir de su comparación con las alternativas disponibles para el realista en el debate acerca de las leyes.

No hay, después de todo, un argumento último para justificar la adopción del RC, ni de la actitud realista que con diferentes matices suele acompañarla. Tampoco es claro -al menos hasta donde puedo vislumbrar- si dicha actitud es motivacional o racionalmente primordial respecto de la afirmación del RC, o viceversa. Estas páginas, sin embargo, han pretendido clarificar parte de sus relaciones mutuas, mostrando qué compromisos relacionados con dicha actitud están fundamentados a partir de la adopción del $\mathrm{RC}$, y en qué sentido esa fundamentación tiene lugar.

\section{Bibliografía}

ARMSTRONG, D. M. “What is a Law of Nature?”. Cambridge: Cambridge University Press, 1983. 
BERENSTAIN, N., LADYMAN, J. "Ontic Structural Realism and Modality". In: E. Landry, D. Ricklets (eds.). Structural Realism: Structure, Object, and Causality. Springer Netherlands, 2012. pp. 149-168.

BORGE, B. "Realismo Científico hoy: a 40 años de la formulación del Argumento del No-Milagro". Acta Scientiarum. Human and Social Sciences, Vol. 37, Nr. 2, pp. 221-233, 2015.

BOYD, R. "On the Current Status of the Issue of Scientific Realism". Erkenntnis, Vol. 19, Nr. 1, pp. 45-90, 1983.

CARROLL, J. W. "Laws of nature". Cambridge: Cambridge University Press, 1994. CARTWRIGHT, N. "How the laws of physics lie". Oxford: Clarendon Press, 1983. CHAKRAVARTTY, A. "A metaphysics for scientific realism: Knowing the unobservable". Cambridge: Cambridge University Press, 2007.

CHISHOLM, R. "The Contrary-to-Fact Conditional”. Mind, Vol. 55, Nr. 1, pp. 289307, 1946.

DRETSKE, F. "Laws of nature". Philosophy of Science, Vol. 44, Nr. 1, pp. 248-268, 1977. ELLIS, B. "Scientific Essentialism". Cambridge: Cambridge University Press, 2001. ENĆ, B. "Reference of theoretical terms". Noûs, Vol. 10, Nr. 3, pp. 261-282, 1976.

FINE, K. "The varieties of necessity". Conceivability and possibility, pp. 253-282, 2002. GOODMAN, N. "The Problem of Counterfactual Conditionals". Journal of Philosophy, Vol. 44, Nr. 1, pp. 113-128, 1947.

HEMPEL, C., OPPENHEIM, P. "Studies in the Logic of Explanation". Philosophy of Science, Vol. 1, Nr. 15, pp. 135-175, 1948.

JACKSON, F. "Reference and description revisited." Nô̂s, Vol. S12, Nr. 32, pp. 201$218,1998$.

KRIPKE, S. "Naming and necessity". In: D. Davidson, H. Harman (eds.). Semantics of Natural Language. Dordrecht, 1972. pp. 334-342.

KROON, F. "Causal descriptivism". Australasian Journal of Philosophy, Vol. 1, Nr. 65, pp. 1-17, 1987.

LADYMAN, J. "What is structural realism?". Studies in History and Philosophy of Science Part A, Vol. 29, Nr. 3, pp. 409-424, 1998.

LEADER, S. "The no miracles argument:" Capture me if you can!" Ma. Dissertation, University of Cape Town, Sudáfrica, 2013.

LEWIS, D. "Philosophical papers, volume II". Oxford: Oxford University Press, 1986. . "Putnam's paradox." Australasian Journal of Philosophy, Vol. 3, Nr. 62, pp. 221-236, 1984.

. "Counterfactuals". Cambridge: Harvard University Press, 1973.

LOEWER, B. "HumeanSupervenience”. Philosophical Topics, Nr. 24, pp. 101-126, 1996. MOLNAR, G. "Powers: A Study in Metaphysics". Oxford: Oxford University, 1996. MONTON, B., VAN FRAASSEN, B. "Constructive Empiricism and Modal Nominalism". The British Journal for the Philosophy of Science, Vol. 54, Nr. 1, pp. 405-422, 2003. MUMFORD, S. "Laws in nature". Cambridge: Routledge, 2004.

NIINILUOTO, I. “Critical Scientific Realism”. Oxford: Oxford University Press, 1999. 
NOLA, R. "Fixing the reference of theoretical terms". Philosophy of Science, Vol. 47, Nr. 4, pp. 505-531, 1980.

PERSSON, J. "Explanation in Metaphysics?". Metaphysica, Vol. 12, Nr. 2, pp. 165$181,2011$.

PRICE, H., CORRY, R. (eds.). "Causation, physics, and the constitution of reality: Russell's republic revisited”. Oxford: Oxford University Press, 2007.

PSILLOS, S. "Regularities, natural patterns and laws of nature". Theoria: Revista de Teoría, Historia y Fundamentos de la Ciencia, Vol. 29, Nr. 79, pp. 9-27, 2014.

. "Causal descriptivism and the reference of theoretical terms." In: A. Raftopoulos,

P. Machamer (eds.). Perception, realism, and the problem of reference. Cambridge: Cambridge University Press, 2012. pp. 212-238.

. "Simply the Best: A Case for Abduction". In: A. Kakas, F. Sadri (eds.). Computational Logic: Logic Programming and Beyond, Essays in Honour of Robert A. Kowalski, Part II, Lecture Notes in Computer Science 2048. Berlin: Springer, 2002. . "Scientific realism: How science tracks truth". New York, London: Routledge, 1999.

PUTNAM, H. "The meaning of "meaning". Minnesota Studies in the Philosophy of Science, Vol. 7, pp. 131-193, 1975a.

. "What is mathematical truth". In: H. Putnam (auth). Philosophical Papers, 1, pp. 69-96, 1975b.

QUINE, W. V. "Main trends in recent philosophy: Two dogmas of empiricism." The Philosophical Review, pp. 20-43, 1951.

RAILTON, P. "Probability, explanation, and information". Synthese, Vol. 2, Nr. 48, pp. 233-256, 1981.

SALMON, W. "Scientific explanation and the causal structure of the world". Princeton: Princeton University Press, 1984.

SHOEMAKER, S. "Causality and properties". Springer Netherlands, 1980.

SOAMES, S. "The philosophical significance of the Kripkean necessary aposteriori." Philosophical Issues, Vol. 16, Nr. 1, pp. 288-309, 2006.

SWARTZ, N. "The Concept of Physical Law". Cambridge: Cambridge University Press, 1985.

TOOLEY, M. "The nature of laws". Canadian Journal of Philosophy, Vol. 7, Nr. 4, pp. 667-698, 1977. 